\title{
A PLL-Based Multirate Structure for Time-Varying Power Systems Harmonic/Interharmonic Estimation
}

\author{
Janison Rodrigues de Carvalho, Carlos A. Duque, Member, IEEE, Moisés V. Ribeiro, Member, IEEE, \\ Augusto S. Cerqueira, Thomas L. Baldwin, Senior Member, IEEE, and Paulo F. Ribeiro, Fellow, IEEE
}

\begin{abstract}
This paper describes a phase-locked-loop (PLL)based power systems harmonic estimation algorithm, which uses an analysis filter bank and multirate processing. The filter bank is composed of bandpass filters. The initial center frequency of each filter is purposely chosen to be equal to harmonic frequencies. However, an adaptation strategy makes it possible to track time-varying frequencies as well as interharmonic components. A downsampler device follows the filtering stage, reducing the computational burden, especially because undersampling operations are performed. Finally, the last stage is composed of a PLL estimator which provides estimates for amplitude, phase, and frequency of the input signal. The proposed method improves the accuracy, computational effort, and convergence time of the previous harmonic estimator based on cascade PLL configuration.
\end{abstract}

Index Terms-Multirate signal processing, phase-locked loop (PLL), time-varying harmonic/interharmonic estimation.

\section{INTRODUCTION}

W ITH the increased application of power electronics, controllers, motor drives, inverters, and flexible ac transmission systems (FACTS) devices in modern power systems, distortions in line voltage and current have been increasing significantly. These distortions have affected the power quality (PQ) of the power system and to maintain it under control, the monitoring of harmonic and interharmonic distortion is an important issue [1]-[3].

The discrete Fourier transform (DFT) is a suitable approach to estimate the spectral content of a stationary signal. However, it loses accuracy under time-varying conditions [4]. As a result, other algorithms must be used. The short-time Fourier transform (STFT) can partly deal with time-varying conditions but it has the limitation of a fixed window width chosen a priori and this imposes limitation for the analysis of low-frequency and highfrequency nonstationary signals at the same time [5].

Manuscript received November 12, 2007; revised September 07, 2008. Current version published September 23, 2009. This work was supported in part by CAPES and in part by CNPq from Brazil. Paper no. TPWRD-00682-2007.

J. R. de Carvalho, M. V. Ribeiro, and A. S. Cerqueira are with the Electrical Engineering Department, Juiz de Fora MG 36036-330, Brazil (e-mail: janison@sel.eesc.usp.br).

C. A. Duque is with the Engineering Faculty at Federal University of Juiz de Fora (UFJF), Juiz de Fora, Brazil 36036-330, and also with the Center for Advanced Power Systems/Florida State University, Tallahassee, FL 32306 USA (e-mail: carlos.duque@ufjf.edu.br).

T. L. Baldwin is with the Center for Advanced Power Systems/Florida State University, Tallahassee, FL 32306 USA (e-mail: tom.baldwin @ ieee.org).

P. F. Ribeiro is with the Center for Advanced Power Systems/Florida State University, Tallahassee, FL 32306 USA, and also with Calvin College, Grand Rapids, MI 49546 USA (e-mail: pfribeiro@ ieee.org).

Digital Object Identifier 10.1109/TPWRD.2009.2027474
The IEC standard drafts [6] have specified signal-processing recommendations and definitions to harmonic and interharmonic measurement. These recommendations utilize the DFT over a rectangular window of exactly 12 cycles for $60 \mathrm{~Hz}(10$ cycles for $50 \mathrm{~Hz}$ ) and a frequency resolution of $5 \mathrm{~Hz}$. However, different authors [7], [8] have shown that the detection and measurement of interharmonics, with acceptable accuracy, are difficult to obtain by using the IEC specification.

Unlike the previous methods that follow the IEC standard, other techniques based on the Kalman filter, adaptive notch filter, or PLL approaches have been applied in harmonic and interharmonic estimation. The main disadvantage of the Kalman filter is the higher order model required to estimate several components [9]. In [3], the enhanced phase-locked loop (EPLL) [2] is used as the basic structure for harmonic and interharmonic estimation, and several of these sections are arranged together. Each one is adjusted to estimate a single sinusoid waveform. The convergence takes about 18 cycles, but it can take more than 100 cycles for higher harmonic frequencies, mostly if there is a fundamental frequency deviation. In [10], a new multirate filter bank structure for harmonic and interharmonic extraction is presented. The method uses EPLL as an estimation tool in combination with sharp bandpass filters and downsampler devices. As a result, an enhanced and low computational complexity method for parameters estimation of time-varying frequency signals is obtained.

This paper presents a new version of the proposed method in [10]. The main contribution of this new version is concerned with the new concept of apparent frequency and the undersampling. The undersampling is deliberately used in order to reduce the computational effort of the overall estimator, while the signal reconstruction remains possible. Furthermore, the convergence time of the parameter estimator is reduced. Finally, but not least, the new approach leads to better structures to be implemented in the fixed-point arithmetic processor. This is because the downsampler feeds the PLL estimator with a fake signal whose frequency is close to $60 \mathrm{~Hz}$. As the PLL parameters are a function of the signal frequency to be estimated, all PLL cores will have a similar structure that can be optimized to be implemented in fixed-point processors. Implementation in fixed-point processors means lower cost of the final product.

This paper is organized as follows. Section II presents some concepts about the digital filter bank. Section III describes the multirate processing, the concepts of undersampling, and apparent frequency. Section IV describes the proposed structure and presents the recursive equations of the EPLL method [2]. Section V presents two structures of harmonic estimation for 


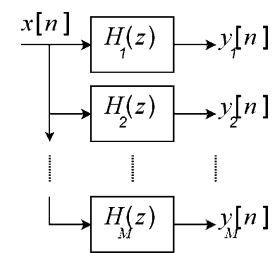

Fig. 1. Typical analysis filter bank.

comparison. The simulation results are shown and discussed in Section VI. Finally, conclusions are stated in Section VII.

\section{Digital Filter BANK}

A digital filter bank [11] is a collection of digital bandpass filters with either a common input (the analysis bank) or a summed output (the synthesis bank). The analysis bank is the focus of this section.

The analysis filter bank decomposes the input signal $x[n]$ into a set of $M$ subband signals $y_{1}[n], y_{2}[n], \ldots, y_{M}[n]$, each one occupying a portion of the original frequency band. Fig. 1 shows a typical analysis filter bank.

In this paper, the filter bank differs from traditional filter banks found in the literature [11], [12]. This is due to the filters employed here. Basically, they are conventional parametric IIR bandpass filters [12] given by

$$
H(z)=\frac{1-\alpha}{2} \cdot \frac{1-z^{-2}}{1-\beta(1+\alpha) z^{-1}+\alpha z^{-2}} .
$$

The main objective of using the bandpass filters consists of extracting each of the components, fundamental and harmonics (or interharmonics), normally found in power systems. Another objective is to reduce the noise level at the input of the estimation stage. This filter was chosen, in this design, for the following reasons:

- it is a parametric filter, which means that the central frequency may be adjusted easily by changing the $\beta$ factor in (1); treating the $\beta$ factor as an input parameter provides a quick way to set the central frequency in an adaptive algorithm;

- at the central frequency, the phase shift induced by the filter is zero; by tuning the central frequency of the IIR filter to the desired bandpass frequency, the signal of interest will not be shifted in phase;

- despite the low order of the IIR bandpass filter (it can used in cascade configuration), the frequency response has an appropriate characteristic.

The magnitude responses of (1) are plotted in Fig. 2(a) for the bandpass centered at a fundamental frequency $(60 \mathrm{~Hz})$ and some of its odd harmonics. Solid lines are for the single second-order filter of (1) while dashed lines show the response for a cascade structure of two second-order filters. The phase response of the filter centered at the fundamental component filter is detailed in Fig. 2(b). Notice the zero-phase response at the frequency 60 $\mathrm{Hz}$ mentioned previously.

The previous transfer function has a narrow bandwidth when the poles are a pair of complex conjugates near the unit circle.

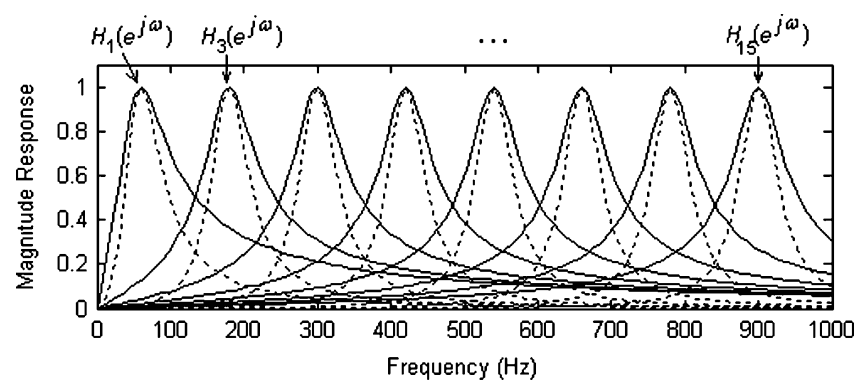

(a)

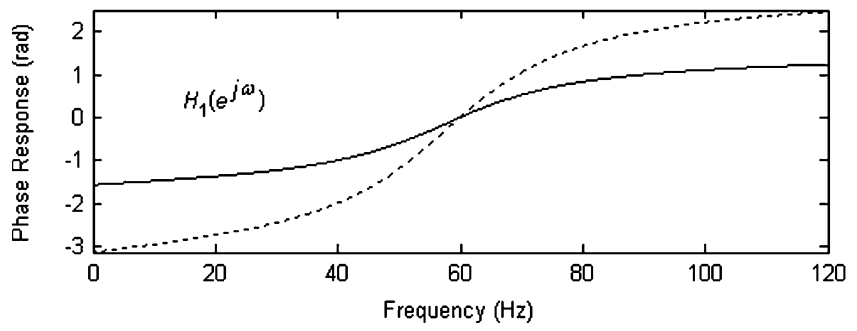

(b)

Fig. 2. Frequency response: (a) Magnitude responses of parametric bandpass filters of (1). (b) Phase response for the filter centered at the fundamental frequency.

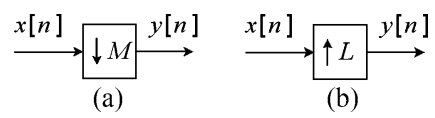

Fig. 3. Block diagram representations of sampling rate alteration devices. (a) The downsampler. (b) The upsampler.

The parameter $\alpha$ controls this proximity defining the $3 \mathrm{~dB}$-bandwith of the filter. The maximum magnitude value occurs at discrete frequency $\omega_{0}$ which is related to $\beta$ by the expression $\beta=$ $\cos \left(\omega_{0}\right)$.

Although the parameter $\alpha$ near the unit produces a sharper magnitude response, it increases the transient time response. This fact is very important because the convergence time of the estimator is proportional to the duration of the transient response caused by the filter. For example, using (1) with $\alpha=0.98$ and an input signal of $60 \mathrm{~Hz}$ with 128 points per cycle, the transient virtually decays at about four cycles.

\section{MultiRAte Processing}

The two basic components in sampling rate modification are: 1) the downsampler, to reduce the sampling rate; and 2) the upsampler, to increase the sampling rate [12]. The block diagram representation of these two components is shown in Fig. 3. The downsampler will be described in this section.

A downsampler with a downsampling factor $M$, where $M$ is a positive integer, creates an output sequence $y[n]$ with a sampling rate $M$ times smaller than the sampling rate of the input sequence $x[n]$.

The input-output relationship can be written as

$$
y[n]=x[n M] .
$$

In frequency domain, it can be shown that [12] 


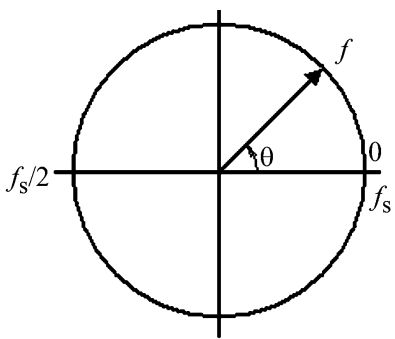

(a)

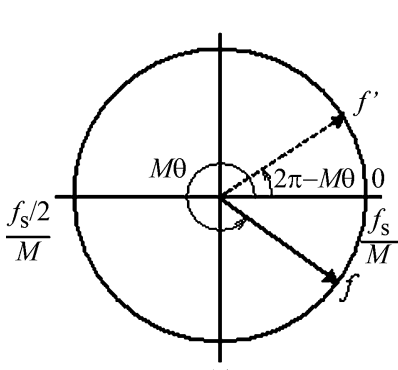

(c)

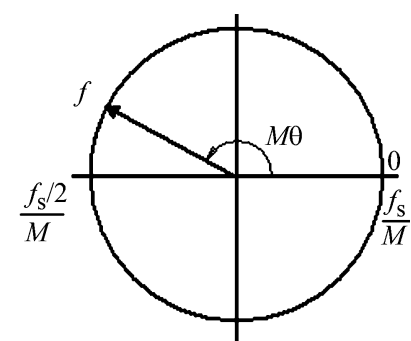

(b)

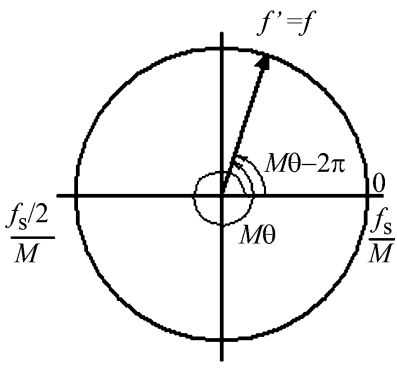

(d)
Fig. 4. Alternative interpretation of the downsample effect in a single sinusoidal signal. (a) Original position of the component with frequency $f$. (b) Position of the component after downsampling $\theta_{M}<\pi$. (c) Position of the component after downsampling $\pi<\theta_{M}<2 \pi$. (d) Position of the component after downsampling $2 \pi<\theta_{M}<3 \pi$.

$$
Y\left(e^{j \omega}\right)=\frac{1}{M} \sum_{k=0}^{M-1} X\left(e^{j(\omega-2 \pi k) / M}\right)
$$

Equation (3) implies that the DTFT of the downsampled output signal $y[n]$ is a sum of $M$ uniformly shifted and stretched versions of DTFT of input $x[n]$, scaled by a factor $1 / M$.

The direct application of (3) is sometimes unnecessary, especially with the assumption of a single sinusoidal component at the downsampler input. A simple method can be applied alternatively, as shown in Fig. 4. Fig. 4(a) shows a circle where a sinusoidal component of frequency $f$ is correctly placed with the angle $\theta$ (in radians) given by

$$
\theta=\frac{f}{f_{S}} \cdot(2 \pi)
$$

where $f_{S}$ is the sampling frequency. After downsampling with a factor $M$, the output component exhibits a new angle position $\theta_{M}=M \cdot \theta$, as shown in Fig. 4(b)-(d).

There are some singularities to be considered when a sinusoid is downsampled. It is clear that undersampling can be performed. As a result, the frequency of the downsampled sequence can be different (lower) from the frequency of the input sequence. This new frequency will be referred to as the apparent frequency. We can resume singularities as:

Case 1) The new angle $\theta_{M}$ is lower than $\pi$ radians. This case is shown in Fig. 4(b). The output signal represents the original signal with frequency $f$, that is, no undersampling was performed.

Case 2) The new angle $\theta_{M}$ falls into the lower semicircle, as shown in Fig. 4(c). The output signal was obtained by undersampling the input and the mirror component, in the dashed line, has to be considered. The apparent frequency $f^{\prime}$ is obtained by analyzing the angle $2 \pi-M \theta$

$$
f^{\prime}=\left(\frac{2 \pi-M \theta}{2 \pi}\right)\left(\frac{f_{S}}{M}\right) .
$$

Case 3) The new angle $\theta_{M}$ falls into the upper semicircle after one revolution, as shown in Fig. 4(d). Undersampling was performed again. The apparent frequency $f^{\prime}$ is obtained by analyzing the angle $M \theta-$ $2 \pi$

$$
f^{\prime}=\left(\frac{M \theta-2 \pi}{2 \pi}\right)\left(\frac{f_{S}}{M}\right) .
$$

If the number of revolutions is greater than 1 , they must be discounted and then case 2 or case 3 can be applied.

The common sense indicates that undersampling (when the sampling frequency is lower than the Nyquist rate) is a prohibitive operation in signal processing, because the aliasing phenomena [12] deforms the signal frequency spectrum. However, there are at least two applications where undersampling is applied and the original signal can be reconstructed without error: 1) in filter bank theory and 2) single sinusoid signal. In the first case, the aliasing phenomena in the filter bank stage is avoided by the proper design of the analysis and synthesis filter banks [11]. The second case is easier to understand and correct, as discussed previously. If the signal is composed by a single sinusoid, the aliasing has no effect in superimposing other components with the component of interest. Thus, the undersampled signal is a representation of the sinusoidal component but with a different frequency - the defined apparent frequency.

\section{Proposed Structure}

The proposed structure for harmonic estimation is shown in Fig. 5. Fifteen bandpass filters compose the filter bank. The $k$ th bandpass filter is previously designed with its central frequency being equal to $k \cdot f_{0}$, where $k=1,2, \ldots, 15$. Although this design is specific to the analysis of harmonic content up to the 15th component, it can be expanded to analyze harmonics of higher order. Basically, the main idea of this research is to show that undersampling can be used in benefit to achieve an efficient and low-cost structure, incorporating the concepts of multirate processing with an estimation tool-the PLL.

After the filtering stage, the downsampler devices reduce the sampling rate, performing an undersampling for $k=5,6, \ldots, 15$, according to Table I. Regarding this table, from $k=5$ to $k=15$, the difference between the true frequency values, placed in column $y_{k}$, and the apparent frequency values, placed in column $v_{k}$ can be noticed.

Finally, the last stage is the estimator stage. This is composed by the Enhanced-PLL (EPLL) system [2], which is responsible for extracting three parameters from its input signal $v_{k}[n]$. These parameters are the magnitude, the frequency, and the total phase, that is

$$
\underline{\hat{\psi}}_{k}[n]=\left[\hat{A}_{k}[n] \hat{\omega}_{k}[n] \hat{\phi}_{k}[n]\right]^{T} .
$$




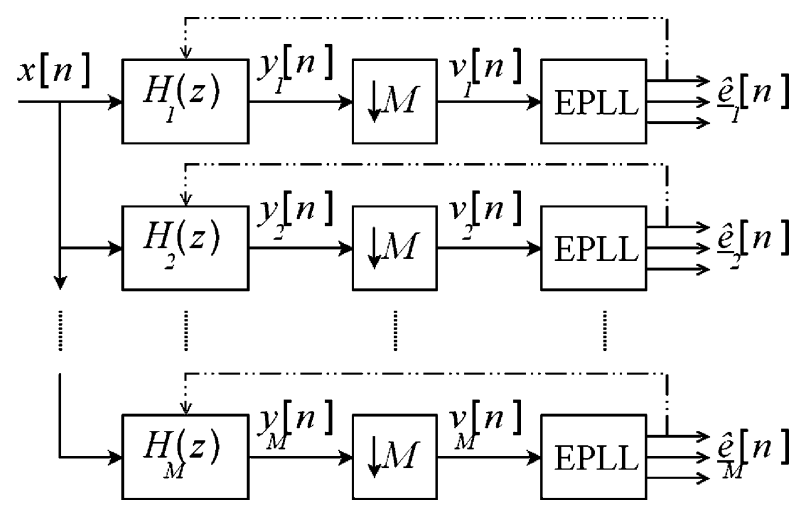

Fig. 5. Proposed structure for harmonic estimation.

TABLE I

TYPICAL VALUES FOR THE DOWNSAMPLING FACTOR AND ITS EFFECTS IN CHANGING FREQUENCY OF THE INPUT SIGNAL

\begin{tabular}{c|c|c|c|c|c|c|c}
\hline \multirow{2}{*}{} & \multirow{2}{*}{$\boldsymbol{M}_{\boldsymbol{k}}$} & \multicolumn{2}{|c|}{ Frequency (Hz) } & \multirow{2}{*}{$\boldsymbol{k}$} & \multirow{2}{*}{$\boldsymbol{M}_{\boldsymbol{k}}$} & \multicolumn{2}{c}{ Frequency (Hz) } \\
\cline { 3 - 7 } & & $\boldsymbol{y}_{\boldsymbol{k}}$ & $\boldsymbol{v}_{\boldsymbol{k}}$ & & & $\boldsymbol{y}_{\boldsymbol{k}}$ & $\boldsymbol{v}_{\boldsymbol{k}}$ \\
\hline $\mathbf{1}$ & 16 & 60 & 60 & $\mathbf{9}$ & 16 & 540 & 60 \\
\hline $\mathbf{2}$ & 8 & 120 & 120 & $\mathbf{1 0}$ & 11 & 600 & 98.18 \\
\hline $\mathbf{3}$ & 16 & 180 & 180 & $\mathbf{1 1}$ & 16 & 660 & 180 \\
\hline $\mathbf{4}$ & 12 & 240 & 240 & $\mathbf{1 2}$ & 12 & 720 & 80 \\
\hline $\mathbf{5}$ & 16 & 300 & 180 & $\mathbf{1 3}$ & 16 & 780 & 180 \\
\hline $\mathbf{6}$ & 14 & 360 & 188.57 & $\mathbf{1 4}$ & 15 & 840 & 184 \\
\hline $\mathbf{7}$ & 16 & 420 & 60 & $\mathbf{1 5}$ & 16 & 900 & 60 \\
\hline $\mathbf{8}$ & 14 & 480 & 68.57 & - & - & - & - \\
\hline
\end{tabular}

The EPLL discrete-time recursive equations are

$$
\begin{aligned}
\hat{A}_{k}[n+1]= & \hat{A}_{k}[n]+\mu_{1} T_{S} e_{k}[n] \sin \left(\hat{\phi}_{k}[n]\right) \\
\hat{\omega}_{k}[n+1]= & \hat{\omega}_{k}[n]+\mu_{2} T_{S} e_{k}[n] \cos \left(\hat{\phi}_{k}[n]\right) \\
\hat{\phi}_{k}[n+1]= & \hat{\phi}_{k}[n]+T_{S} \hat{\omega}_{k}[n] \\
& +\mu_{3} T_{S} e[n] \cos \left(\hat{\phi}_{k}[n]\right)
\end{aligned}
$$

where $\mu_{1}, \mu_{2}$, and $\mu_{3}$ are constants that determine the speed of convergence and steady-state error, $T_{S}$ is the sampling period, and $e_{k}[n]$ is the error signal given by

$$
e_{k}[n]=v_{k}[n]-\hat{A}_{k}[n] \sin \left(\hat{\phi}_{k}[n]\right) .
$$

From Fig. 5, it can be seen that the estimated frequency of each EPLL block is used to update its respective bandpass filter. There are many strategies to do this. Here, we chose to use an instantaneous update by using the output of a moving average filter (MAF) attached to the EPLL block output.

\section{A. Theoretical Analysis of the Multirate Structure}

Signal enhancement is a common preprocessing approach used in parameter estimation. It consists of extracting an unwanted signal component or noise from the source signal that would degrade the estimation performance. When used as an estimator, the EPLL shows improvements when signal enhancement is performed first. The supporting theoretical argument comes from the periodic orbit theorem associated with the EPLL dynamic equations [13].

First, assume an electrical signal is composed of the fundamental, several harmonics components, and noise. This signal can be written as

$$
x(t)=x_{0}(t)+x_{h}(t)+n(t)
$$

where $x_{0}(t)$ is the fundamental component, $x_{h}(t)$ is the sum of harmonics, and $n(t)$ is the noise. Let the objective be the extraction of the $x_{0}(t)$ component. The theorem concludes that the response of dynamic equations of EPLL will converge to a periodic solution in the neighborhood of $x_{0}(t)$. This occurs for a proper choice of parameters $\mu_{i}\{i=1,2,3\}$ and the neighborhood is determined by these parameters and the component $x_{h}(t)+n(t)$. In other words, if the fundamental component of a highly polluted electrical signal is to be extracted, the estimation will present oscillations due to the presence of harmonics and noise. When using analysis filter banks (for signal enhancement), the energy $x_{h}(t)+n(t)$ is reduced, so the periodic orbit becomes close to $x_{0}(t)$.

This theorem can be extended to the estimation of each harmonic and to the case when the frequency is time varying as stated below.

Assume that the $k$ th bandpass filter was designed with a central frequency equal to $k f_{0}$ and there is a frequency deviation $\left(k\left(f_{0}+\Delta f\right)\right)$. Despite the sharp magnitude response of the bandpass filter (Fig. 2), the component close to $k f_{0}$ is not completely attenuated and the value of the frequency, phase, and amplitude of it will be estimated. The capability of EPLL of tracking the actual frequency of the attenuated component is used to update the central frequency of the bandpass filter and, after the transient response, all parameters are correctly estimated, since the phase response of the filter is zero at the central frequency. The smaller the frequency deviation is (such as in electric power systems), the faster the transient response is.

Note that all other frequency components than the central frequency are attenuated by the filter, which reduces the term $x_{h}(t)$ in (10). Furthermore, the bandpass filter increases the signal-to-noise ratio (SNR) (i.e., it reduces the noise variance) and consequently $n(t)$, by a factor that depends on the parameter $\alpha$. The theoretical relationship between the output variance $\sigma_{o}^{2}$ and input variance $\sigma_{i}^{2}$ is given by [12]

$$
\sigma_{o}^{2}=\frac{\sigma_{i}^{2}}{2 \pi} \int_{-\pi}^{\pi}\left|H_{k}\left(e^{j \omega}\right)\right|^{2} d \omega .
$$

For example, for $\alpha=0.98$, the SNR is increased by $17 \mathrm{~dB}$.

Thus, the analysis filters are used to split the components, improving PLL performance by providing ("theoretically") a single sinusoidal signal at the input of each PLL.

\section{B. Downsampling Benefit}

The advantages of inserting the downsamplers between the bandpass filter and the EPLL estimators are twofold: 1) it reduces the computational complexity of the algorithm and 2) it allows the definition of a single set of EPLL parameters, applied uniformly to each frequency band. The first advantage is 
based on exchanging the extra operations from the filtering stage with the simplification of the estimation stage by using a lower sampling rate. The second advantage comes from a single set of EPLL constants. In a single-rate approach (conventional), these constants must be modified and adjusted for each harmonic component. In a multirate approach, the EPLL estimates an undersampled signal whose frequency is between $60-240 \mathrm{~Hz}$ (see Table I) and only one set of constants is necessary. This strategy is adopted to allow the parameter estimation of any harmonic in a frequency band where the EPLL constants were exhaustively tested. This fact explains the reasons for the choice of the downsampler factors in Table I.

\section{METHODS FOR COMPARISON}

A brief description of two methods used for comparison is made here. Two parameters are used for comparison with these methods. The first one is the convergence time, defined here as the time necessary to attain and stay inside the $\pm 2 \%$ error band. The second one is the mean squared error (MSE) on steady state. For any parameter $p$, taken from (8), the MSE is given by

$$
\operatorname{MSE}(p)=\frac{1}{N} \sum_{n=0}^{N-1}\left(p[n]-p_{0}\right)^{2}
$$

where $N$ is the number of samples and $p_{0}$ is the actual value.

\section{A. Short-Time Fourier Transform (STFT)}

The STFT is also known as the time-dependent Fourier transform. Let $x[n]$ be the signal of interest. The STFT of $x[n]$ is defined as [12]

$$
X_{\mathrm{STFT}}[k, n]=\sum_{m=0}^{R-1} x[n-m] w[m] e^{-j 2 \pi k m / N}
$$

where $w[n]$ is a suitably chosen window of length $R$ and $k=$ $0,1,2, \ldots, R-1$ is the harmonic order.

Interpreting (13), the STFT of $x[n]$ at time $n=n_{0}$ is the DFT of the sequence $x\left[n_{0}-m\right] w[m]$. Shifting $n$ from $n_{0}$ to $n_{1}$, the STFT is now the DFT of the sequence $x\left[n_{1}-m\right] w[m]$. It can be concluded that the STFT provides the spectral contents of the sliding window in time of the sequence $x[n]$.

The choice of window sequence depends on desirable characteristics of each one in the frequency domain, such as a reduction of leakage effect, etc. [14]. Some examples of windows are: Rectangular, Hamming, Hann, and Bartlett.

\section{B. EPLL Cascade Structure}

This method was proposed in [3]. It employs a cascade configuration of modified units of (8), as is shown in Fig. 6. The signal to be analyzed is the input of the unit responsible to extract the fundamental component $y_{1}[n]$. This component is subtracted from the input signal and then the second unit is responsible to extract the second harmonic component. This process continues until the $k$ th component of interest.

The EPLL blocks on Fig. 6 are said to be modified because of the use of two first-order lowpass filters on their internal structure. These filters are included to prevent the error on estimating

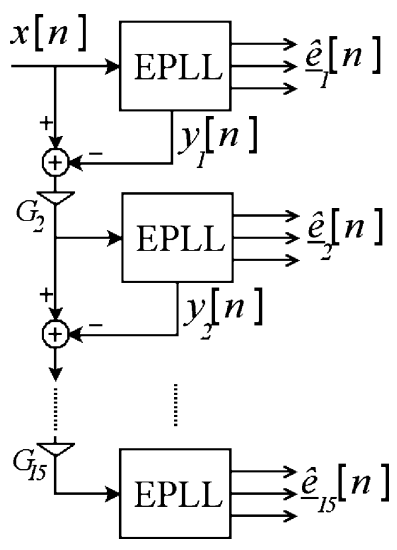

Fig. 6. EPLL cascade structure proposed in [3] for harmonic and interharmonic estimation.

the component of interest caused by the other harmonic and interharmonics components and noise. Besides, first-order lowpass filters are also included on the output to smooth results.

\section{Comments on Asynchronous Sampling}

Although the EPLL cascade structure performance does not depend on the sampling process, the STFT performance is directly related to this process. If the sampling rate is supposed to be constant and synchronous with the fundamental component, and only the harmonic component is presented in the signal, the STFT becomes the better fit for harmonic estimation. However, the STFT is not able to track frequency changes, and provides only the amplitude and phase angle of the $k$ th component by calculating the modulus and phase of the complex number of (13). With asynchronous sampling, it loses accuracy, as discussed in the next section.

The STFT can be interpreted as an analysis filter bank composed of complex filters. Assuming a frequency deviation in an electrical signal under analysis, a possible way of adapting the STFT is to provide synchronous sampling. Thus, the central frequency of the $k$ th filter of (13) is adapted to match the actual frequency. We can observe a similar strategy in [15] where a variable sampling frequency is presented to improve the phasor estimation for digital relaying.

Fig. 7 shows the magnitude response of the STFT filter, with a rectangular window centered at $60 \mathrm{~Hz}$. The magnitude response for the cascade filter consisting of two second-order bandpass filters (1) is shown in the same figure for comparison.

Note that the STFT has high rejection for the harmonic frequencies. If the sampling rate is not synchronous or if there are interhamonic components in the input signal, then the estimation error increases significantly. On the other hand, when compared with the STFT, the bandpass filter presents better noise rejection over all frequencies, except at the harmonic frequencies. Furthermore, the update of the central frequency of the bandpass filter is easily accomplished because the filter is parametric.

In this paper, the STFT was implemented without employing a synchronization algorithm and using a Hamming window, being used as an additional comparison method. However, it is worth mentioning that there is a lack of works in the literature 


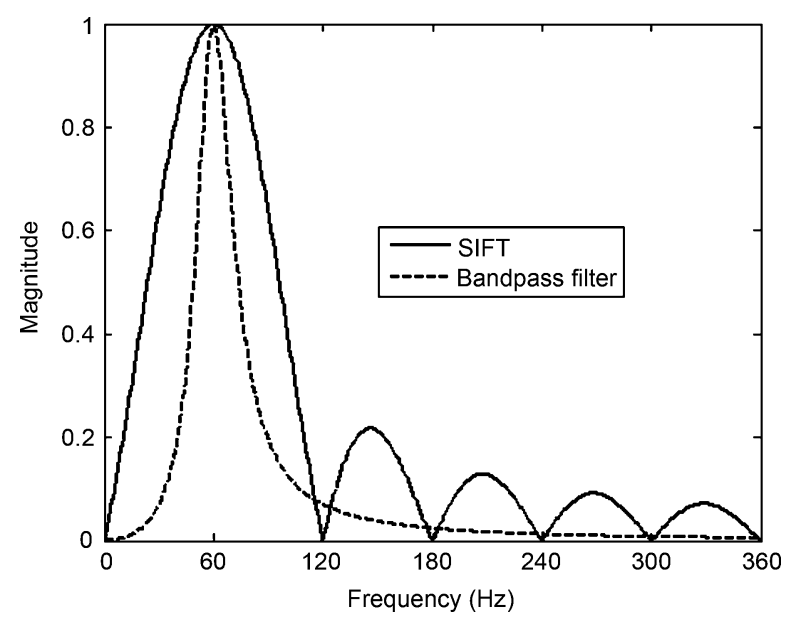

Fig. 7. Magnitude response of STFT and bandpass filter centered at $60 \mathrm{~Hz}$.

that addresses the STFT under the time-varying frequency condition.

\section{Simulations Results}

This section presents the performance of the proposed method for some commonly found conditions of parameter variations of the power system voltage signal. All simulations were performed by programming all methods in MATLAB. The simulation parameters are the following. The sampling frequency is $f_{S}=128.60 \mathrm{~Hz}$, that is, 128 points per cycle of a signal with frequency $f_{0}=60 \mathrm{~Hz}$. A cascade structure of two bandpass filters of (1) is used with $\alpha=0.98$. The EPLL constants $\mu_{1}, \mu_{2}$, and $\mu_{3}$ are 300,500 , and 6 , respectively, for all units. The order of MAF filters used at the EPLL output is 24 . The process of determining the set of constants starts with the use of constants of previous works [1]-[3], [13]. Particularly, in [2], some good tips are given to solve this question. Following the instruction of [13], the set used was determined after a large battery of numerical tests. We observed low transient response (about 1 cycle) and low steady-state error.

The STFT method uses the Hamming window of length 128, that is, one cycle of the fundamental signal. For the EPLL cascade structure, the constants set are $\mu_{1}=100, \mu_{2}=700$, and $\mu_{3}=50$ for the fundamental component and increases with the order of component. For the 15th harmonic component, the constants are $\mu_{1}=100, \mu_{2}=1300$, and $\mu_{3}=75$. These values for constants are higher than that used in [3] with the objectives of the estimations becoming more fast.

We emphasize that frequency deviations and harmonic levels considered here are nonrealistic in real power system voltage signals. However, these deviations and levels are commonly used in the literature to test measurement systems to emphasize its performance in severe conditions of variation. For example, with a step changing in frequency, it is interesting to observe the time of convergence of the methodology.

\section{A. Presence of Harmonics}

In this case, a typical power system polluted signal is considered, with the signal corrupted by harmonics. The amplitudes

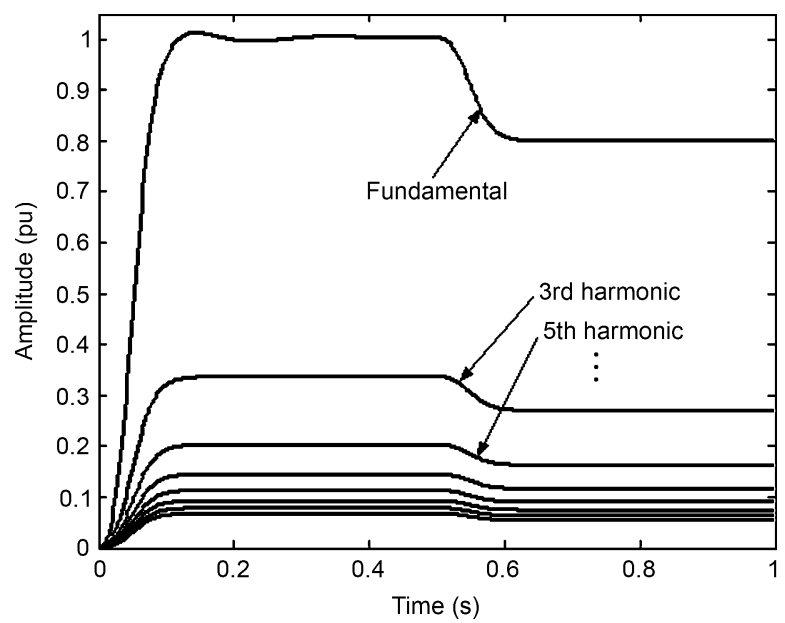

Fig. 8. Amplitude estimation: presence of harmonics and disturbance in amplitude at time $t=0.5 \mathrm{~s}$.

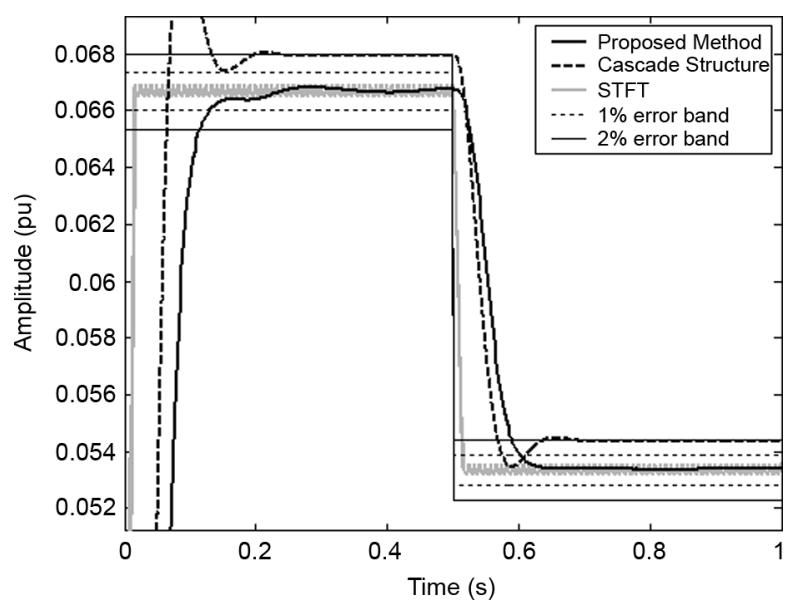

Fig. 9. Comparison of methods for estimating the 15th harmonic.

of harmonics are inversely proportional to its order. Besides, at time $t=0.5 \mathrm{~s}$, the amplitude is abruptly reduced

$$
\begin{aligned}
x(t)=V_{M} \sin \left(2 \pi f_{0} t\right)+ & \sum_{\substack{k=3 \\
\text { odd }}}^{15} \frac{1}{k} V_{M} \sin \left(2 \pi k f_{0} t\right) \\
& \left\{\begin{array}{ll}
V_{M}=1.0 & 0 \leq t<0.5 \\
V_{M}=0.8 & t \geq 0.5
\end{array} .\right.
\end{aligned}
$$

Fig. 8 shows the estimated amplitudes of (14). The method is capable of extracting each component. Amplitude estimation of the 15th harmonic component is detailed in Fig. 9. The results of cascade structure and STFT method are also shown for comparison. Note that the result of multirate structure remains inside \% error band after transient response, as well as the result of the STFT method. However, for cascade structure, the estimation is very close to the edge of $2 \%$ error band. This behavior is observed for the other harmonic components.

The MSE and convergence time after disturbance can be observed, respectively, in Fig. 10(a) and (b). The multirate structure presented MSE errors lower than the cascade structure and convergence time was about $0.1 \mathrm{~s}$ for all components. The STFT 


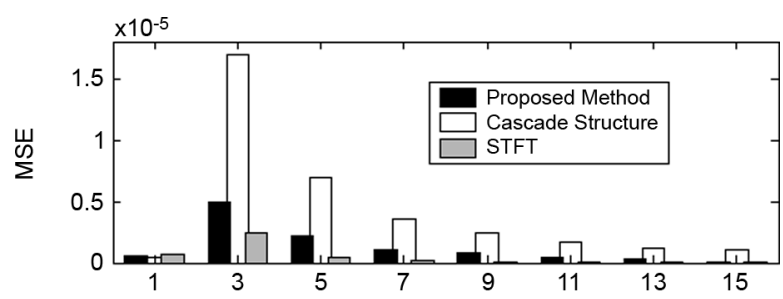

(a)

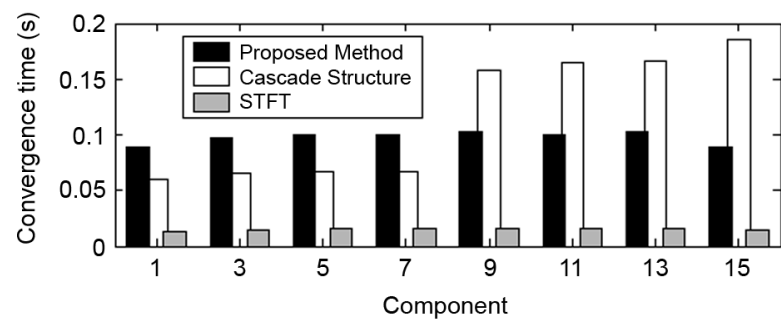

(b)

Fig. 10. Parameters of comparison for the input signal of (14), after the disturbance occurred at $t=0.5 \mathrm{~s}$. (a) Mean-squared error. (b) Convergence time.

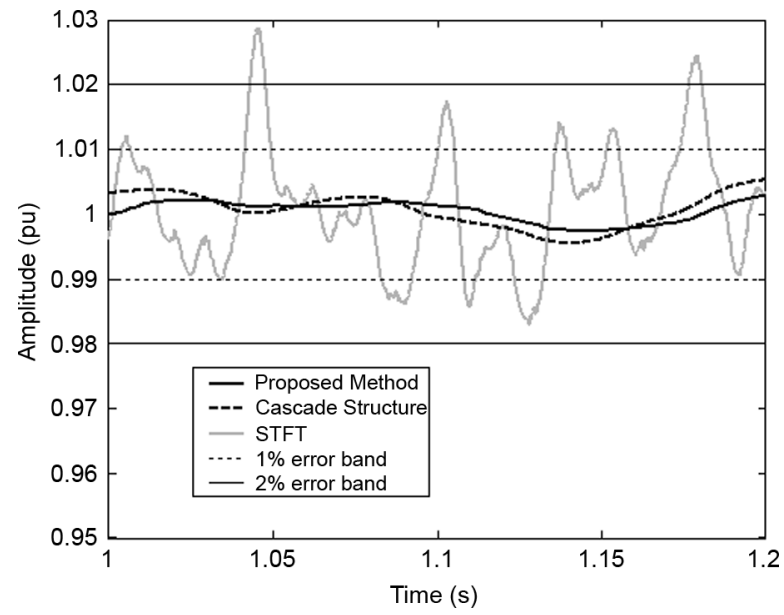

Fig. 11. Amplitude estimation for $\mathrm{SNR}=17.5 \mathrm{~dB}$.

presents the lowest MSE and it is the fastest method, since there is no frequency deviation.

\section{B. Presence of Additive White Gaussian Noise}

This case tests the noise immunity of the multirate method. A zero-mean white Gaussian noise $n(t)$, with variance $\sigma^{2}$, is added to the fundamental component to obtain the input signal

$$
x(t)=V_{M} \sin \left(2 \pi f_{0} t\right)+n(t)
$$

where $n(t)=N\left(0, \sigma^{2}\right)$.

Fig. 11 presents results of simulations for an SNR of $17.5 \mathrm{~dB}$. The proposed method and cascade structure present better results than STFT.

Comparison of methods is made regarding Fig. 12. It shows the relationship between MSE and SNR for the three methods. It can be seen that the multirate structure presents the best performance for an input signal corrupted by additive noise.

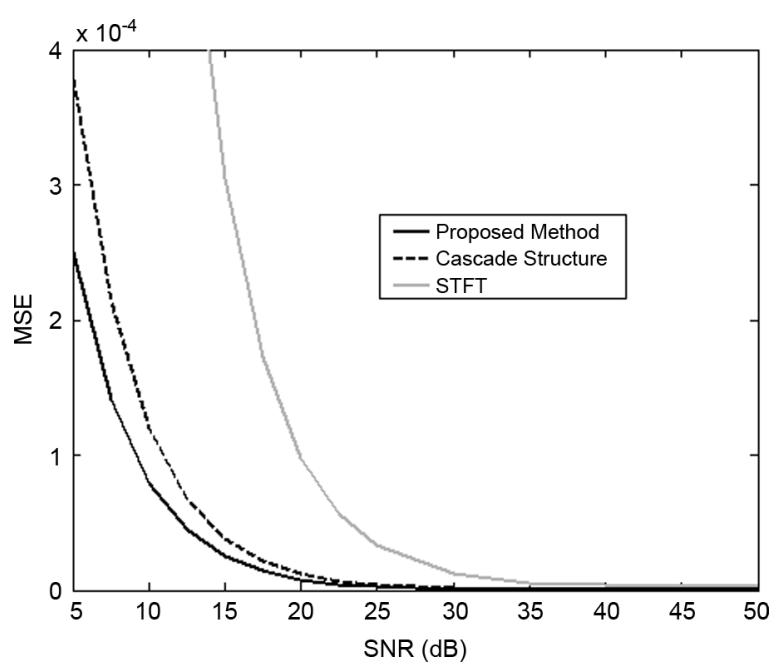

Fig. 12. Relationship between MSE and SNR for the three methods considered.

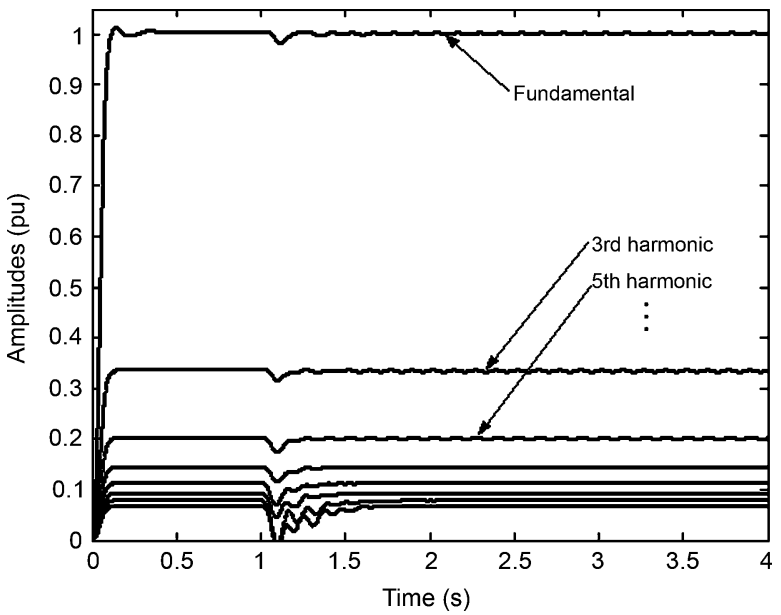

Fig. 13. Amplitude estimation: Effect of step changing in frequency.

\section{Step Variation of Frequency}

In this case, a disturbance in frequency is forced. The input signal is also composed of odd harmonics but at time $t=1 \mathrm{~s}$, a step change in fundamental frequency is imposed

$$
\begin{gathered}
x(t)=V_{M} \sin \left(2 \pi f_{0} t\right)+\sum_{\substack{k=3 \\
\text { odd }}}^{15} \frac{1}{k} V_{M} \sin \left(2 \pi k f_{0} t\right) \\
\left\{\begin{array}{ll}
f_{0}=60 & \leq t<1.0 \\
f_{0}=61 & t \geq 1.0
\end{array} .\right.
\end{gathered}
$$

Fig. 13 shows the estimated amplitudes of (16). Note that the step changing in frequency causes a disturbance in amplitude estimation. However, the system is capable of providing correct results, after a transient response.

The amplitude estimation of the 11th harmonic component is detailed in Fig. 14. The STFT method fails for this input signal condition, presenting an instantaneous error that reaches $4.3 \%$ for this harmonic. The multirate and cascade structures 


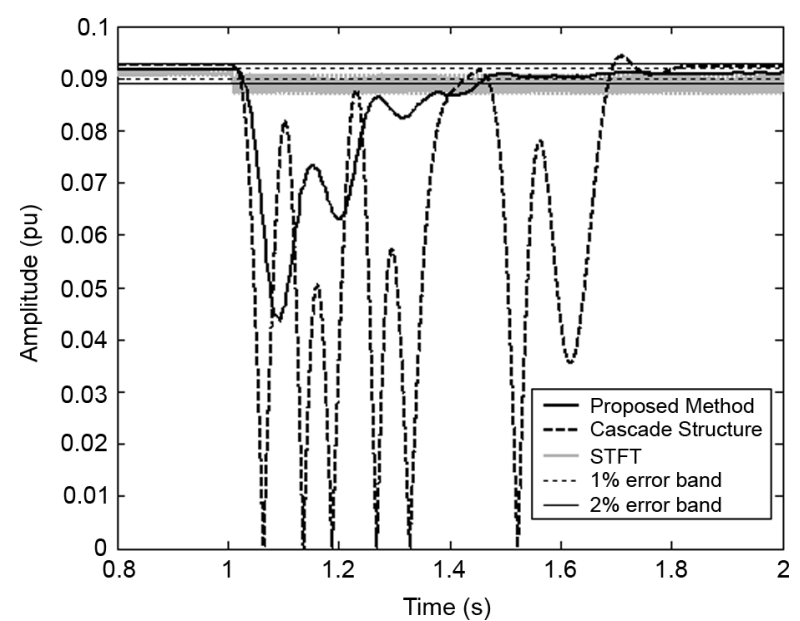

Fig. 14. Comparison of methods for estimating the 11th harmonic.

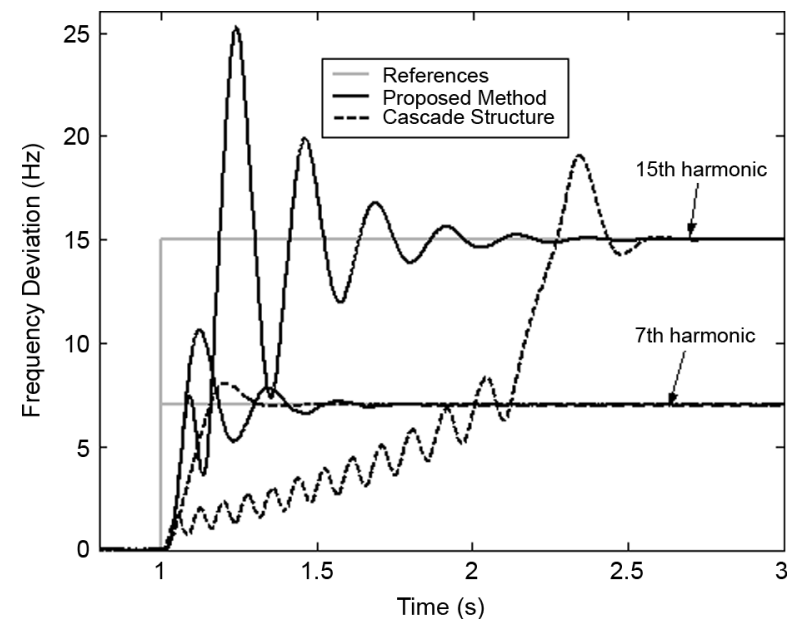

Fig. 15. Frequency tracking of the 7 th and 15 th harmonics for a step change in fundamental frequency $\left(\Delta f_{0}=1 \mathrm{~Hz}\right)$.

provide correct estimations. Note that the multirate structure is the fastest to attain the $2 \%$ error band after the disturbance.

Since $f_{0}$ jumps from 60 to $61 \mathrm{~Hz}$, the frequency deviation of the fundamental component is $\Delta f_{0}=1 \mathrm{~Hz}$. So, the frequency deviation of the $k$ th harmonic is $\Delta f_{k}=k \mathrm{~Hz}$. The frequency tracking characteristics of the 7th and 15th harmonic components are shown in Fig. 15. Note that the larger the frequency deviation is, the longer the time to track it. This fact explains the longest transient time of high-order components in Fig. 13.

The MSE for three methods is shown in Fig. 16(a). Unlike the previous cases, STFT presents a large error. The multirate structure keeps its performance presenting the smallest MSE. The convergence time of amplitude after the disturbance is shown in Fig. 16(b). This parameter is not computed for the STFT method because the estimation is not limited to the $2 \%$ error band. Note that the proposed method is faster than the cascade structure.

\section{Sinusoidal Variation of Frequency}

A more realistic case is evaluated now. The input signal is considered to have a sinusoidal variation of frequency around

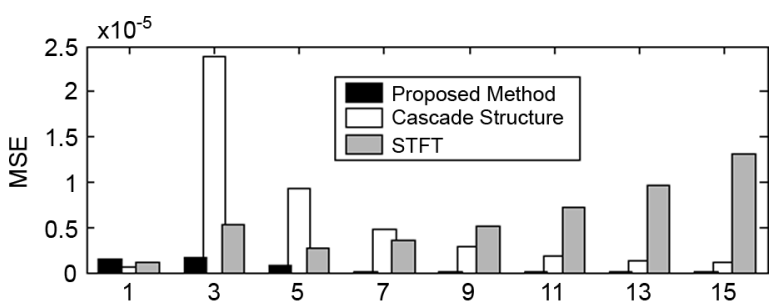

(a)

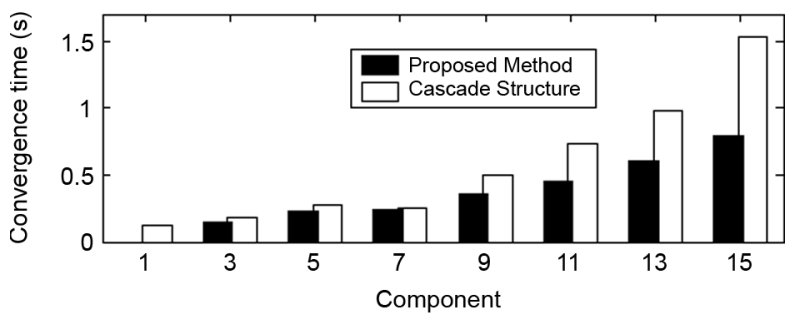

(b)

Fig. 16. Parameters of comparison for the input signal of (16), after the disturbance occurred at $t=1 \mathrm{~s}$. (a) Mean-squared error. (b) Convergence time.

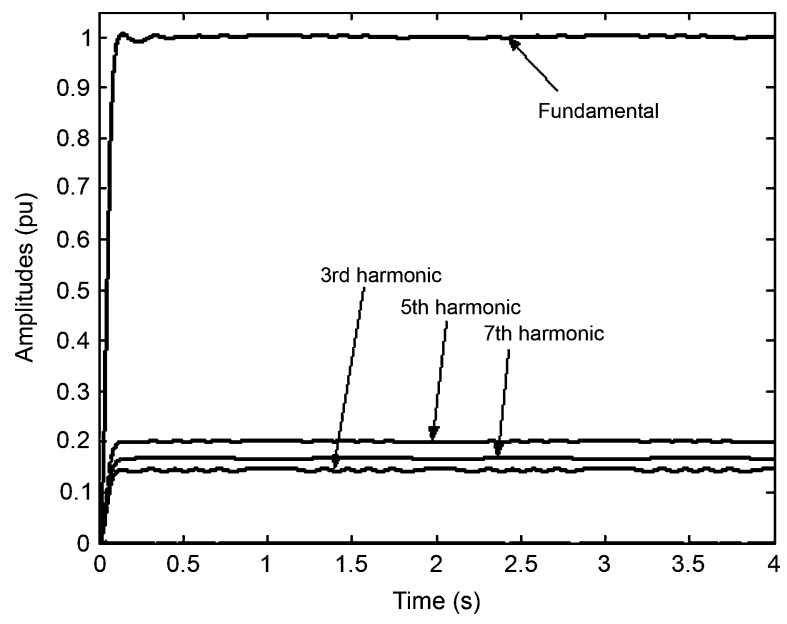

Fig. 17. Amplitude estimation: sinusoidal variation of frequency of input signal (17).

the ideal value $f_{0}$. The $3 \mathrm{rd}, 5$ th, and 7 th harmonic components are also present in the input signal

$$
\begin{aligned}
x(t)=V_{M}[ & \sin (\phi(t))+\left(\frac{1}{7}\right) \sin (3 \phi(t)) \\
& \left.+\left(\frac{1}{5}\right) \sin (5 \phi(t))+\left(\frac{1}{6}\right) \sin (7 \phi(t))\right] .
\end{aligned}
$$

The total phase $\phi(t)$ is given by

$$
\phi(t)=\int_{0}^{t}\left[f_{0}+\Delta f_{0} \sin \left(2 \pi f_{F} t\right)\right] d t
$$

where $\Delta f_{0}$ is the maximum frequency deviation and $f_{F}$ is the frequency of variation. Fig. 17 shows estimated amplitudes of (17) considering $\Delta f_{0}=1 \mathrm{~Hz}$ and $f_{F}=0.5 \mathrm{~Hz}$.

Details of amplitude estimation for the 5th harmonic component are shown in Fig. 18. Note that in this case, the cascade 


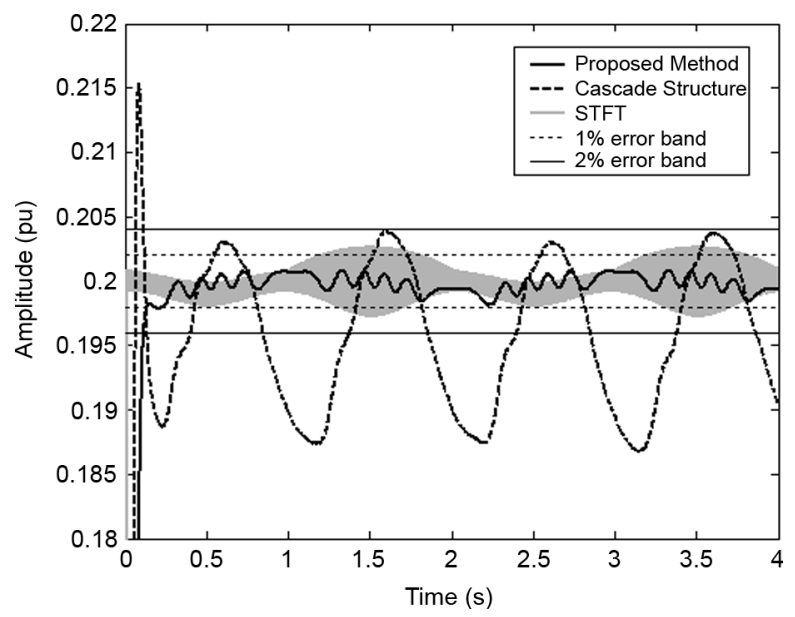

Fig. 18. Comparison of methods for estimating the 5th harmonic.

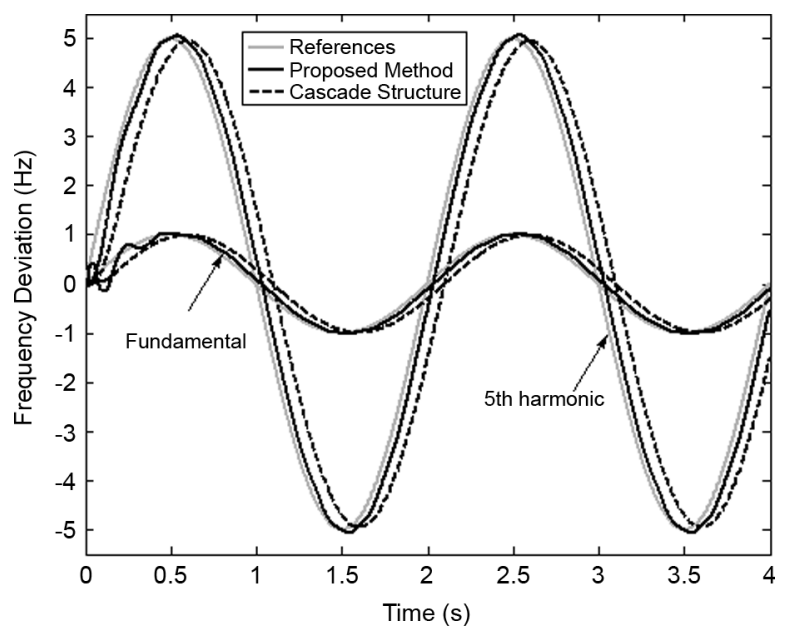

Fig. 19. Frequency tracking of the fundamental and 5th harmonic components for sinusoidal variation.

structure fails. The multirate structure provides amplitude estimation for this component within the $1 \%$ error band. STFT presents an oscillatory behavior that tends to increase in proportion to $\Delta f_{0}$.

The frequency tracking performance is shown in Fig. 19 for the fundamental and 5th harmonic components. The proposed method provides results closer to the references than the cascade structure. The delays in time of estimations are $30 \mathrm{~ms}$ and 100 $\mathrm{ms}$ for the multirate method and cascade structure, respectively.

The MSE of the estimated amplitudes is shown in Fig. 20. The proposed method provides low MSE for every component of the input signal.

\section{E. Ramp Variation of Frequency}

In this case, a ramp variation of frequency is considered. This is a common condition found in a load-shedding scenario. The 3rd and 5th harmonics are also present in the input signal

$x(t)=V_{M} \sin (\phi(t))+\frac{V_{M}}{3} \sin (3 \phi(t))+\frac{V_{M}}{5} \sin (5 \phi(t))$.

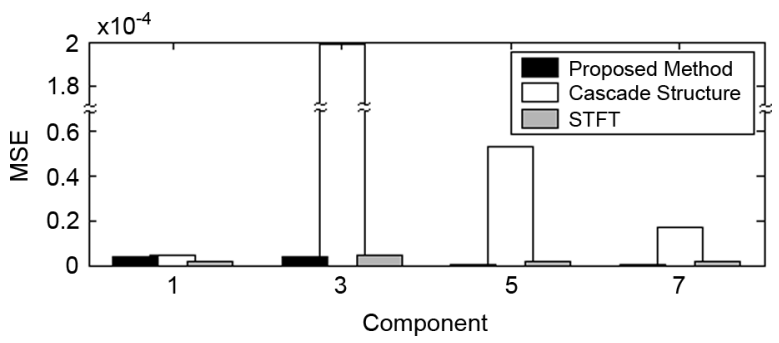

Fig. 20. Parameter of comparison for the input signal of (17), the mean-squared error.

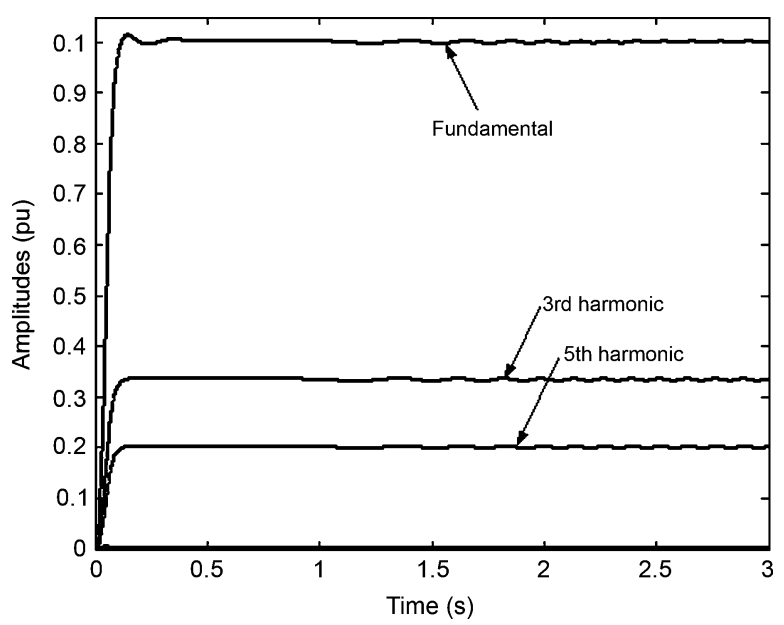

Fig. 21. Amplitude estimation: effect of ramp variation of frequency.

The total phase $\phi(t)$ is given by

$$
\phi(t)=\int_{0}^{t} f(t) \mathrm{d} t
$$

where the instantaneous frequency $f(t)$ is

$$
f(t)= \begin{cases}f_{0} & 0 \leq t<t_{i} \\ f_{0}+\frac{\Delta f_{0}}{t_{f}-t_{i}}\left(t-t_{i}\right) & t_{i} \leq t<t_{f} \\ f_{0}+\Delta f_{0} & t \geq t_{f}\end{cases}
$$

In (21), $\Delta f_{0}$ is the frequency deviation and $t_{i}$ and $t_{f}$ are the beginning and the end of the ramp, respectively. Fig. 21 shows the estimated amplitudes for the input signal of (19) considering $\Delta f_{0}=1 \mathrm{~Hz}, t_{i}=0.75 \mathrm{~s}$, and $t_{f}=2.25 \mathrm{~s}$. That is, a ramp variation of frequency is considered within the interval $0.75 \leq$ $t<2.25$ with a slope of $0.667 \mathrm{~Hz} / \mathrm{s}$.

Details of amplitude estimation for the fifth harmonic component are shown in Fig. 22. Note that for STFT, the instantaneous error increases in proportion to the increase in frequency. The multirate structure provides amplitude estimation for this component within the $1 \%$ error band.

The frequency tracking performance is shown in Fig. 23 for all components. Both methods are able to track the ramp variation imposed in frequency. 


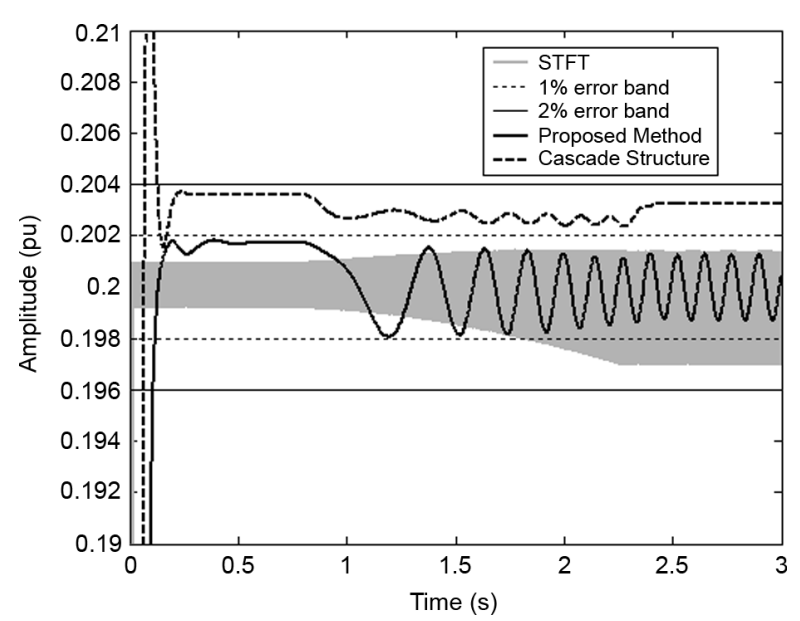

Fig. 22. Comparison of methods for estimating the 5th harmonic.

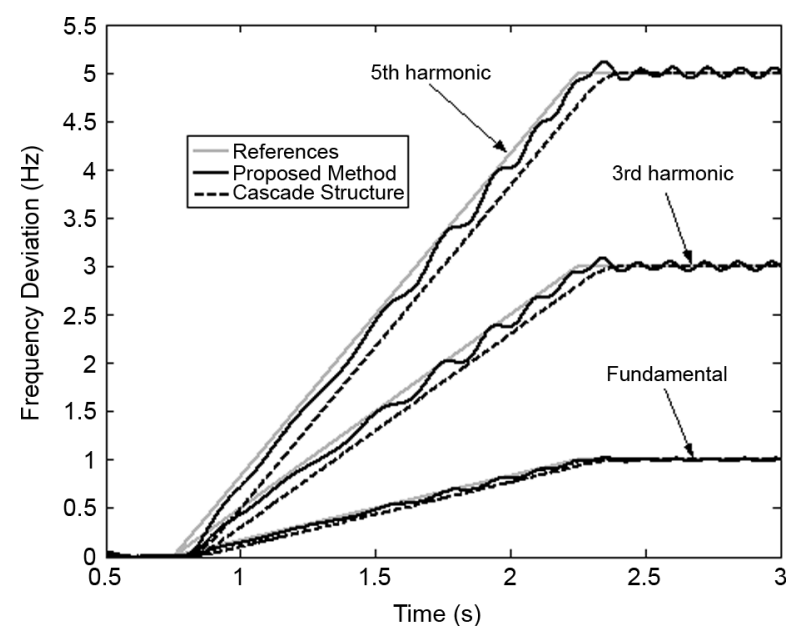

Fig. 23. Frequency tracking for the fundamental, 3rd, and 5th harmonic components.

\section{F. Presence of the Interharmonic Component}

Finally, this case tests the capability of estimating an interharmonic component. The input signal is considered to be composed of the fundamental and the interharmonic component

$$
x(t)=V_{M} \sin \left(2 \pi f_{0} t\right)+\frac{V_{M}}{5} \sin \left(2 \pi f_{I} t\right)
$$

where $f_{I}=200 \mathrm{~Hz}$ is the interharmonic frequency considered for simulation.

Fig. 24 shows the amplitude estimations for components of (22). Note the increase in convergence time for the amplitude of interharmonics compared to the case of harmonic components. This is due to the initialization of the structure with harmonic frequencies.

Amplitude estimation of the interharmonic component is detailed in Fig. 25. The result of the cascade structure is shown for comparison while STFT is not able to track this component because of the window length. The difference between the convergence of the two methods is evident. Convergence time of the proposed structure is $0.44 \mathrm{~s}$ against $19.2 \mathrm{~s}$ of the cascade

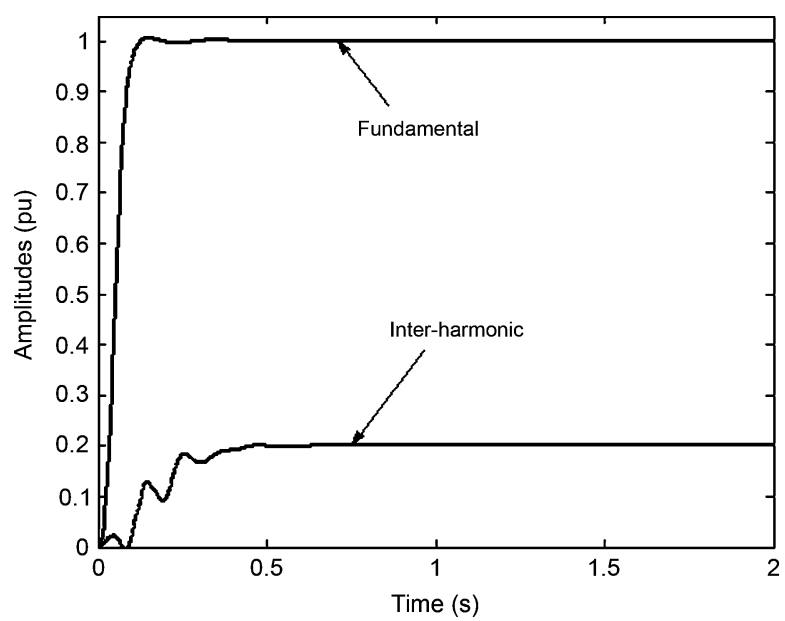

Fig. 24. Amplitude Estimation: presence of an interharmonic frequency $f_{I}=$ $200 \mathrm{~Hz}$.

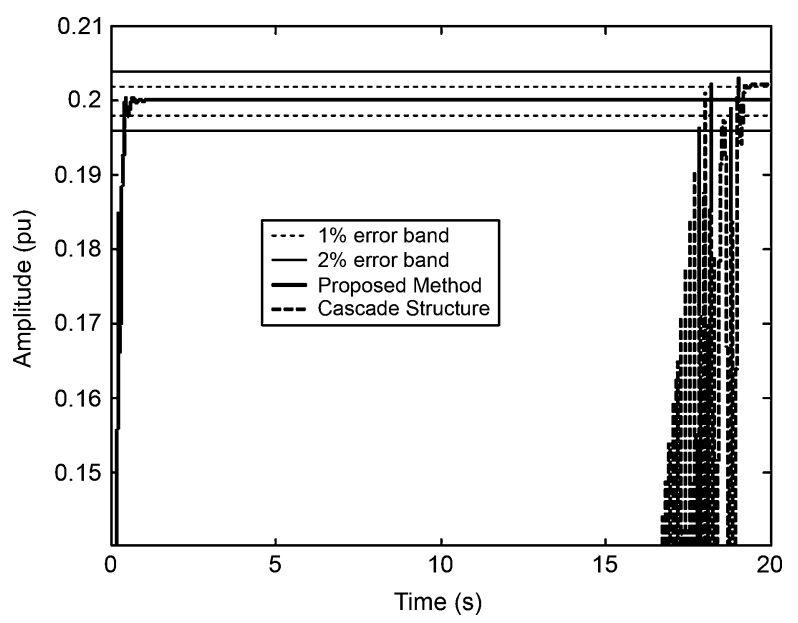

Fig. 25. Comparison of methods for estimating the interharmonic component.

structure. Besides, the multirate method provides amplitude estimation within the $1 \%$ error band.

Fig. 26 shows the frequency tracking for the interharmonic component. Note the capability of quickly tracking the multirate structure.

\section{G. Phase Estimation}

Phase estimation is an important parameter for the phasor measurement unit (PMU) and control of active filters. Besides, it is an important parameter to characterize the electrical power system in general terms. However, when the system frequency is time varying, it is a challenging task. The proposed method is able to correct the phase when the frequency changes. The worst case is when the frequency varies according to (18). This case will be considered in this section.

For simulation, we assume constant phases $\theta$ for each component of (17). Thus, the total phase for the $k$ th harmonic component is given by

$$
\phi_{k}=k\left(\int_{0}^{t} \omega(\tau) d \tau\right)+\theta_{k}
$$




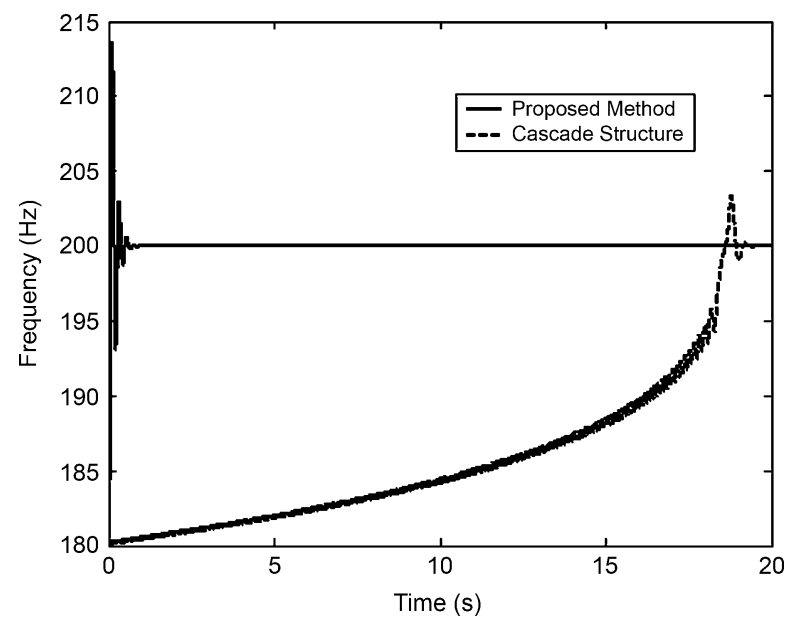

Fig. 26. Frequency tracking of the interharmonic component.

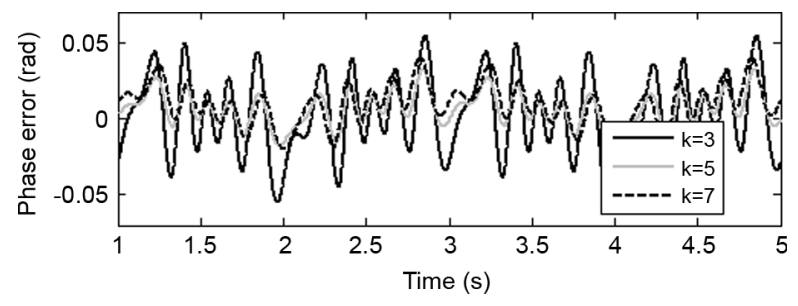

Fig. 27. Error in the estimation of constant phase $\theta$.

TABLE II

MSE- PHASE ERROR COMPARISON

\begin{tabular}{l|c|c|c}
\hline \multirow{2}{*}{$\begin{array}{c}\text { Estimation } \\
\text { Method }\end{array}$} & \multicolumn{3}{|c}{ MSE } \\
\cline { 2 - 4 } & $\mathbf{3}^{\text {rd }}$ harmonic & $\mathbf{5}^{\text {th }}$ harmonic & $\mathbf{7}^{\text {th }}$ harmonic \\
\hline $\begin{array}{c}\text { Cascade EPLL } \\
\text { Structure }\end{array}$ & 0.1536 & 0.0035 & 0.0642 \\
\hline $\begin{array}{l}\text { Multirate } \\
\text { Structure }\end{array}$ & 0.0232 & 0.0153 & 0.0178 \\
\hline
\end{tabular}

Let $\theta_{k}$ be, respectively. equal to $0,0.8,0.5$, and -1.5 radians for $k=1,3,5$, and 7 . The estimated phase is given by

$$
\hat{\theta}_{k}=\hat{\phi}_{k}-k \hat{\phi}_{1}
$$

where $\phi_{k}$ is the harmonic estimated total phase $(k=3,5$, and 7 ), including the constant phase term. Note that the fundamental total phase is used as a reference.

Fig. 27 shows the phase error, given by

$$
\Delta \hat{\theta}_{k}=\hat{\theta}_{k}-\theta_{k} .
$$

As observed, the maximum error is smaller than $0.06 \mathrm{rad}$, which is the same order than the sample angle which is given by $2 \pi /(128)=0.0491$ (128 is the number of sample per cycle). Note that the third harmonic presented the worst error due the proximity with the $60-\mathrm{Hz}$ component.

Table II shows the MSE for the proposed method and the Cascade EPLL structure. The MSE for the STFT is not shown because the STFT is not able to estimate the phase in this case. Note that the cascade EPLL has better phase estimation only for the 5th harmonic.
TABLE III

COMPUTATIONAL EFFort IN NUMBER OF AdDS, Multiplications, AND TABLE SEARCh (FunCtions Sin E COS)

\begin{tabular}{c|c|c|c}
\hline Estimation Method & \multicolumn{3}{|c}{ Operations } \\
\cline { 2 - 4 } & Adds & multiplications & table search \\
\hline $\begin{array}{c}\text { Cascade EPLL } \\
\text { Structure }\end{array}$ & 24960 & 30720 & 3840 \\
\hline $\begin{array}{c}\text { STFT Method } \\
\text { (Hamming window) }\end{array}$ & 489600 & 986880 & 491520 \\
\hline $\begin{array}{c}\text { Multirate } \\
\text { Structure }\end{array}$ & 13438 & 15495 & 411 \\
\hline
\end{tabular}

\section{H. Computational Complexity Comparison}

The simulations have shown advantages of the proposed method in terms of convergence time and steady-state error. The computational effort is evaluated now. It is computed by the number of multiplications, additions, and table search (functions sin and $\cos$ ) for each method: the multirate method, the EPLL-cascade structure, and the STFT method.

Table III shows the number of operations performed in one cycle of the fundamental component to estimate from this component to the 15th harmonic component, including the even order harmonics. This table shows superiority of the multirate structure. Compared with operations of the cascade structure, the proposed method performs only $53.8 \%$ of additions, $50.4 \%$ of multiplications, and $10.7 \%$ of table search. Compared with the STFT method that uses a square root operation not computed in Table III, the advantages of using the proposed method are more evident. Although the inclusion of bandpass filters increases the number of operations, the undersampling operation compensates these extra numbers of additions, multiplications, and table searches. Thus, a low computational effort structure is obtained.

\section{Hardware Implementation}

Although all simulations in this paper were performed by using float-point arithmetic, it is worth mentioning that the multirate structure is useful for fixed-point arithmetic implementation. This is because the EPLL cores track the apparent frequency, instead off the real harmonic. As we can see in Table I, the highest apparent frequency to track is $240 \mathrm{~Hz}$. This means that all cores can be configured with the same internal parameters. This regularity is highly desirable in fixed-point implementation.

\section{CONCLUSION}

This paper presents an improved PLL-based structure for time-varying harmonic and interharmonic estimation. This structure makes use of multirate signal processing. The main objective is to show how analytical filter banks can be applied together with an estimation tool (EPLL) for PQ monitoring applications and identifying the advantages of using it.

The STFT, with a rectangular window, is the best estimation approach if the power system frequency is not time varying and there is not interharmonics in the input signal. However, if these conditions fail, an additional circuit must be included to guarantee a coherent sampling rate and a better window must be 
used in order to attenuate the interharmonic component. For example, by using the Hamming window, better results than the traditional DFT method are obtained when a frequency deviation occurs, but the interharmonic measurement is possible only if STFT is applied on multiple cycles of the fundamental signal. This paper shows that the computational effort for a nonrectangular window is higher than the multirate approach.

The EPLL-cascade structure is capable of frequency tracking and is able to estimate interharmonic, but this structure presented the highest convergence time. This fact is, in part, due to the internal filters added to the PLL structure. These filters also create difficulty for the constants adjustment to track the components. The multirate structure is able to track interharmonic and improves the harmonic estimation in the presence of the interharmonic by enhancing the input signal that will be processed by the EPLL. However it does not solve the problem of interharmonic completely and more research must be carried out in this direction.

The theoretical proof of convergence of the present algorithm is out of the scope of this paper; however, with the bandpass update strategy adopted here, all simulations examples converged in a small time when compared with other PLL approaches. The challenge now is reducing the convergence time and in this case, new strategies of update must be investigated.

Finally, by comparing these two methods - the multirate structure and the cascade of EPLL - the former provides fast estimations with low steady-state error, especially due to the prefiltering stage. Besides, the undersampling operation allows the extraction of parameters of high-order harmonics with a low sampling rate. This operation is responsible for significantly reducing the computational effort and reducing the difficulty of implementing the method in digital signal processor (DSP)-based systems.

\section{REFERENCES}

[1] M. Karimi-Ghartemani and M. R. Iravani, "A nonlinear adaptive filter for online signal analysis in power systems: Applications," IEEE Trans. Power Del., vol. 17, no. 2, pp. 617-622, Apr. 2002.

[2] M. Karimi-Ghartemani and M. R. Iravani, "Robust and frequency-adaptive measurement of peak value," IEEE Trans. Power Del., vol. 19, no. 2, pp. 481-489, Apr. 2004.

[3] M. Karimi-Ghartemani and M. R. Iravani, "Measurement of harmonics/interharmonics of time-varying frequencies," IEEE Trans. Power Del., vol. 20, no. 1, pp. 23-31, Jan. 2005.

[4] Y. Baghzouz, R. F. Burch, A. Capasso, A. Cavallini, A. E. Emanuel, M. Halpin, A. Imece, A. Ludbrook, G. Montanari, K. J. Olejniczak, P. Ribeiro, S. Rios-Marcuello, L. Tang, R. Thaliam, and P. Verde, "Time-varying harmonics: Part I-Characterizing measured data," IEEE Trans. Power Del., vol. 13, no. 3, pp. 938-944, Jul. 1998.

[5] M. V. Chilukuri and P. K. Dash, "Multiresolution S-transform-based fuzzy recognition system for power quality events," IEEE Trans. Power Del., vol. 19, no. 1, pp. 323-329, Jan. 2004.

[6] Electromagnetic compatibility (EMC)_Part 4-7: Testing and measurement techniques-General guide on harmonics and interharmonics measurements and instrumentation, for power supply systems and equipment connected thereto, IEC Std. 61000-4-7, 2002, Ed. 2.0.

[7] D. Gallo, R. Langella, and A. Testa, "Desynchronized processing technique for harmonic and interharmonic analysis," IEEE Trans. Power Del., vol. 19, no. 3, pp. 993-1001, Jul. 2004.

[8] L. L. Lai, C. T. Tse, W. L. Chan, and A. T. P. So, "Real-time frequency and harmonic evaluation using artificial neural networks," IEEE Trans. Power Del., vol. 14, no. 1, pp. 52-59, Jan. 1999.

[9] H. Ma and A. A. Girgis, "Identification and tracking of harmonic sources in a power system using a Kalman filter," IEEE Trans. Power Del., vol. 11, no. 3, pp. 1659-1665, Jul. 1996.
[10] J. R. Carvalho, P. H. Gomes, C. A. Duque, M. V. Ribeiro, A. S. Cerqueira, and J. Szczupak, "PLL based multirate harmonic estimation," in Proc. IEEE Power Eng. Soc. General Meeting, 2007, pp. 1-6.

[11] P. P. Vaidyanathan, Multirate Systems and Filter Bank. Englewood Cliffs, NJ: Prentice-Hall, 1993, pp. 151-157.

[12] S. K. Mitra, Digital Signal Processing: A Computer-based Approach, 3rd ed. New York: McGraw-Hill, 2006, pp. 383-385.

[13] M. Karimi-Ghartemani and A. K. Ziarani, "Periodic orbit analysis of two dynamical systems for electrical engineering applications," J. Eng. Math., vol. 45, pp. 135-154, Feb. 2003.

[14] F. H. Harris, "On the use of windows for harmonic analysis using the discrete Fourier transform," Proc. IEEE, vol. PROC-66, no. 1, pp. 51-83, Jan. 1978.

[15] G. Benmouyal, "An adaptive sampling-interval generator for digital relaying," IEEE Trans. Power Del., vol. 4, no. 3, pp. 1602-1609, Jul. 1989.

Janison Rodrigues de Carvalho was born in Cataguases, Brazil, on July 27, 1980. He received the B.Sc. degree in electrical engineering from Universidade Federal de Viçosa (UFV), Viçosa, Brazil, in 2006 and the M.Sc. degree in electrical engineering from Universidade Federal de Juiz de Fora (UFJF), Juiz de Fora, Brazil, in 2008. He is beginning his studies in the Electrical Engineering D.Sc. Program at the Universidade de São Paulo (USP), Escola de Engenharia de São Carlos- SP (EESC).

His interests include digital signal processing and power quality.

Carlos A. Duque (M'91) was born in Juiz de Fora, Brazil, in 1962. He received the B.S. degree in electrical engineering from the Federal University of Juiz de Fora (UFJF), Juiz de Fora, Brazil, in 1986, and the M.Sc. and Ph.D. degrees in electrical engineering from the Catholic University of Rio de Janeiro, Rio de Janeiro, Brazil, in 1990 and 1997, respectively.

Since 1989, he has been a Professor in the Engineering Faculty at the UFJF in Brazil. His research interests include power-quality analysis, digital instrumentation, and digital signal processing.

Moisés V. Ribeiro (S'03-M'05) was born in Três Rios, Brazil, in 1974. He received the B.S. degree in electrical engineering from the Federal University of Juiz de Fora (UFJF), Juiz de Fora, Brazil, in 1999, and the M.Sc. and Ph.D. degrees in electrical engineering from the University of Campinas (UNICAMP), Brazil, in 2001 and 2005, respectively.

Currently, he is an Assistant Professor at UFJF. His research interests include computational intelligence, digital and adaptive signal processing, power quality, power-line communication, and digital communications.

Augusto S. Cerqueira received the B.S. and D.Sc. degrees in electrical engineering at the Federal University of Rio de Janeiro, Rio de Janeiro, Brazil, in 1998 and 2002, respectively.

He has been an Associate Professor in the Electrical Engineer Department of the Federal University of Juiz de Fora, Juiz de Fora, Brazil, since 2004. He has been working recently with instrumentation, digital signal processing, and power-quality issues.

Thomas L. Baldwin (S'86-M'92-SM'01) received the B.S.E.E. and M.S.E.E. degrees from Clemson University, Clemson, SC, in 1987 and 1989, respectively, and the Ph.D. degree in electrical engineering from Virginia Polytechnic Institute and State University, Blacksburg, in 1993.

Currently, he is an Associate Professor at the Florida State University (FSU) College of Engineering at FSU, Tallahassee, and a Research Engineer at the Center for Advanced Power Systems. His research is in power distribution system design, industrial power systems, grounding issues, transformers, and the analysis of power-quality problems.

Paulo F. Ribeiro (M'78-SM'88-F'03) received the B.S. degree in electrical engineering from the Universidade Federal de Pernambuco, Recife, Brazil and the Ph.D. degree from the University of Manchester Institute of Science and Technology, Machester, U.K.

Currently, he is a Professor of Engineering at Calvin College, Grand Rapids, MI, on sabbatical at the Center for Advanced Power Systems (CAPS)/ Florida State University, Tallahassee. 